International Journal of Applied Mathematics

Volume 34 No. $2 \quad 2021,363-376$

ISSN: 1311-1728 (printed version); ISSN: 1314-8060 (on-line version)

doi: http://dx.doi.org/10.12732/ijam.v34i2.12

\title{
ON HYPERBOLIC-PARABOLIC PROBLEMS WITH INVOLUTION AND NEUMANN BOUNDARY CONDITION
}

\author{
Maksat Ashyraliyev \\ Department of Mathematics, Bahcesehir University \\ Istanbul - 34353, TURKEY
}

\begin{abstract}
We study a nonlocal boundary value problem and a space-wise dependent source identification problem for one-dimensional hyperbolic-parabolic equation with involution and Neumann boundary condition. The stability estimates for the solutions of these two problems are established. The first order of accuracy stable difference schemes are constructed for the approximate solutions of the problems under consideration. Numerical results for two test problems are provided.
\end{abstract}

AMS Subject Classification: 65N06, 35M10, 35R30

Key Words: hyperbolic-parabolic equation; involution; nonlocal boundary value problem; source identification problem; difference scheme; stability

\section{Introduction}

The theory and applications of local and nonlocal problems for mixed type partial differential equations have been investigated by many scientists (see, e.g., [12], [16], [18] and the references given therein). In particular, the theory of nonlocal boundary value problems for hyperbolic-parabolic equations and the numerical methods for their approximate solutions have been the subject of recent research (see, e.g., [3], [4] and the references therein).

Partial differential equations with unknown source terms are used in mathe-

Received: December 20, 2020

(C) 2021 Academic Publications 
matical modeling of real-life systems in different fields of science and technology. In the study of so-called direct problems, the solution of a differential equation is realized by means of (non)local initial and/or boundary conditions, while in inverse problems the equation itself is also unknown. The determination of both the governing equation and its solution requires imposing some conditions additional to those in the corresponding direct problem. The theory of inverse source identification problems for partial differential equations has been developed in great detail over many decades (see, e.g., [14], [15] and the references therein). In recent years, the first attempts have been made to study the source identification problems for hyperbolic-parabolic equations and the corresponding difference schemes for their approximate solutions (see [2], [9], [10], [11]). In this ongoing research, a particular attention is given recently to the source identification problem for hyperbolic-parabolic equation with involution and Dirichlet boundary condition (see [1]). Note that partial differential equations with involution have been recently investigated in the context of direct problems (see [5], [6], [7], [13]). However, the theory of inverse source identification problems for partial differential equations with involution has not been well developed yet.

The present paper is devoted to the study of source identification problems for hyperbolic-parabolic differential and difference equations with involution and Neumann boundary condition. The study of inverse source identification problems is usually based on the reduction of these problems to corresponding direct problems with nonlocal conditions, and therefore, we consider additionally a nonlocal boundary value problems for hyperbolic-parabolic differential and difference equations with involution and Neumann boundary condition. The stability of these problems is established. Numerical results are presented. 


\section{Stability of Differential Equations}

First, we consider the following nonlocal boundary value problem:

$$
\left\{\begin{array}{c}
u_{t t}(t, x)-\left(a(x) u_{x}(t, x)\right)_{x}-\beta\left(a(-x) u_{x}(t,-x)\right)_{x} \\
\quad+\delta u(t, x)=f(t, x),-\ell<x<\ell, 0<t<1, \\
u_{t}(t, x)-\left(a(x) u_{x}(t, x)\right)_{x}-\beta\left(a(-x) u_{x}(t,-x)\right)_{x} \\
\quad+\delta u(t, x)=g(t, x),-\ell<x<\ell,-1<t<0, \\
u_{x}(t,-\ell)=u_{x}(t, \ell)=0,-1 \leq t \leq 1, \\
u\left(0^{+}, x\right)=u\left(0^{-}, x\right), u_{t}\left(0^{+}, x\right)=u_{t}\left(0^{-}, x\right),-\ell \leq x \leq \ell, \\
u(-1, x)=\sum_{j=1}^{P} \alpha_{j} u\left(\lambda_{j}, x\right)+\varphi(x),-\ell \leq x \leq \ell,
\end{array}\right.
$$

for one-dimensional hyperbolic-parabolic differential equation with involution and Neumann boundary condition. Throughout this paper, we assume that the following conditions hold:

$$
\begin{gathered}
\bar{a} \geq a(x)=a(-x) \geq \underline{a}>0, x \in(-\ell, \ell), \underline{a}-\bar{a}|\beta| \geq 0, \\
\sum_{j=1}^{P}\left|\alpha_{j}\right| \leq 1, \quad-1<\lambda_{1} \leq \cdots \leq \lambda_{P} \leq 1 .
\end{gathered}
$$

Under compatibility conditions, nonlocal boundary value problem (1) has a unique smooth solution $u(t, x)$ for the given smooth functions $a(x), \varphi(x), f(t, x)$, $g(t, x)$ and positive constant $\delta$.

Here and in the rest of this paper, let the Sobolev space $W_{2}^{2}[-\ell, \ell]$ be defined as the set of all functions $v(x)$ defined on $[-\ell, \ell]$ such that both $v(x)$ and $v^{\prime \prime}(x)$ are locally integrable in $L_{2}[-\ell, \ell]$, equipped with the norm

$$
\|v(x)\|_{W_{2}^{2}[-\ell, \ell]}=\left(\int_{-\ell}^{\ell}|v(x)|^{2} d x\right)^{1 / 2}+\left(\int_{-\ell}^{\ell}\left|v^{\prime \prime}(x)\right|^{2} d x\right)^{1 / 2} .
$$

Throughout this paper, we denote by $M$ the positive constants which are not expected to be evaluated. We write $M(\delta)$ to emphasize that the constant $M$ depends only on $\delta$.

Theorem 1. Suppose that $\varphi \in W_{2}^{2}[-\ell, \ell]$. Let function $f(t, x)$ be continuously differentiable in $t$ on $[0,1] \times[-\ell, \ell]$ and function $g(t, x)$ be continuously 
differentiable in $t$ on $[-1,0] \times[-\ell, \ell]$. Then, the solution of the nonlocal boundary value problem (1) satisfies the following stability estimates

$$
\begin{gathered}
\|u\|_{C\left([-1,1], L_{2}[-\ell, \ell]\right)} \\
\leq M_{1}(\delta)\left[\|\varphi\|_{L_{2}[-\ell, \ell]}+\|f\|_{C\left([0,1], L_{2}[-\ell, \ell]\right)}+\|g\|_{C\left([-1,0], L_{2}[-\ell, \ell]\right)}\right], \\
\|u\|_{C^{(2)}\left([0,1], L_{2}[-\ell, \ell]\right)}+\|u\|_{C^{(1)}\left([-1,0], L_{2}[-\ell, \ell]\right)}+\|u\|_{C\left([-1,1], W_{2}^{2}[-\ell, \ell]\right)} \\
\leq M_{2}(\delta)\left[\|\varphi\|_{W_{2}^{2}[-\ell, \ell]}+\|f\|_{C^{(1)}\left([0,1], L_{2}[-\ell, \ell]\right)}+\|g\|_{C^{(1)}\left([-1,0], L_{2}[-\ell, \ell]\right)}\right] .
\end{gathered}
$$

Proof. Problem (1) can be written as the abstract nonlocal boundary value problem

$$
\left\{\begin{array}{l}
u^{\prime \prime}(t)+A u(t)=f(t), 0<t<1, \\
u^{\prime}(t)+A u(t)=g(t), \quad-1<t<0 \\
u\left(0^{+}\right)=u\left(0^{-}\right), u^{\prime}\left(0^{+}\right)=u^{\prime}\left(0^{-}\right), \\
u(-1)=\sum_{j=1}^{P} \alpha_{j} u\left(\lambda_{j}\right)+\varphi
\end{array}\right.
$$

in a Hilbert space $L_{2}[-\ell, \ell]$ with self-adjoint positive definite operator $A=A^{x}$ defined by the formula

$$
A^{x} u(x)=-\left(a(x) u_{x}(x)\right)_{x}-\beta\left(a(-x) u_{x}(-x)\right)_{x}+\delta u(x)
$$

with the domain $D\left(A^{x}\right)=\left\{u \in W_{2}^{2}[-\ell, \ell] \mid u^{\prime}(-\ell)=u^{\prime}(\ell)=0\right\}$. Here, $f(t)=$ $f(t, x)$ and $g(t)=g(t, x)$ are given abstract functions, $u(t)=u(t, x)$ is unknown function. The proof of Theorem 1 is based on the theorem on stability of nonlocal abstract problem (4) (see [3]), the self-adjointness and positive definiteness of the space operator $A^{x}$ defined by formula (5) (see [6]).

Second, we consider the following space-wise dependent source identification 
problem

$$
\left\{\begin{array}{l}
u_{t t}(t, x)-\left(a(x) u_{x}(t, x)\right)_{x}-\beta\left(a(-x) u_{x}(t,-x)\right)_{x} \\
\quad+\delta u(t, x)=p(x)+f(t, x), \quad-\ell<x<\ell, 0<t<1, \\
u_{t}(t, x)-\left(a(x) u_{x}(t, x)\right)_{x}-\beta\left(a(-x) u_{x}(t,-x)\right)_{x} \\
\quad+\delta u(t, x)=p(x)+g(t, x),-\ell<x<\ell,-1<t<0, \\
u\left(0^{+}, x\right)=u\left(0^{-}, x\right), u_{t}\left(0^{+}, x\right)=u_{t}\left(0^{-}, x\right),-\ell \leq x \leq \ell, \\
u_{x}(t,-\ell)=u_{x}(t, \ell)=0,-1 \leq t \leq 1, \\
u(-1, x)=\varphi(x), u(1, x)=\psi(x),-\ell \leq x \leq \ell
\end{array}\right.
$$

for one-dimensional hyperbolic-parabolic differential equation with involution and Neumann boundary condition. Under assumption (2) and compatibility conditions, problem (6) has a unique smooth solution $(u(t, x), p(x))$ for the given smooth functions $a(x), \varphi(x), \psi(x), f(t, x), g(t, x)$ and constant $\delta>0$.

Theorem 2. Suppose that $\varphi, \psi \in W_{2}^{2}[-\ell, \ell]$. Let function $f(t, x)$ be continuously differentiable in $t$ on $[0,1] \times[-\ell, \ell]$ and function $g(t, x)$ be continuously differentiable in $t$ on $[-1,0] \times[-\ell, \ell]$. Then, the solution of the identification problem (6) satisfies the stability estimates

$$
\begin{gathered}
\|u\|_{C\left([-1,1], L_{2}[-\ell, \ell]\right)}+\left\|\left(A^{x}\right)^{-1} p\right\|_{L_{2}[-\ell, \ell]} \leq M_{3}(\delta)\left[\|\varphi\|_{L_{2}[-\ell, \ell]}\right. \\
\left.+\|\psi\|_{L_{2}[-\ell, \ell]}+\|f\|_{C\left([0,1], L_{2}[-\ell, \ell]\right)}+\|g\|_{C\left([-1,0], L_{2}[-\ell, \ell]\right)}\right] \\
\|u\|_{C^{(2)}\left([0,1], L_{2}[-\ell, \ell]\right)}+\|u\|_{C^{(1)}\left([-1,0], L_{2}[-\ell, \ell]\right)}+\|u\|_{C\left([-1,1], W_{2}^{2}[-\ell, \ell]\right)} \\
+\|p\|_{L_{2}[-\ell, \ell]} \leq M_{4}(\delta)\left[\|\varphi\|_{W_{2}^{2}[-\ell, \ell]}+\|\psi\|_{W_{2}^{2}[-\ell, \ell]}\right. \\
\left.+\|f\|_{C^{(1)}\left([0,1], L_{2}[-\ell, \ell]\right)}+\|g\|_{C^{(1)}\left([-1,0], L_{2}[-\ell, \ell]\right)}\right]
\end{gathered}
$$

Proof. Problem (6) can be written in the following abstract form

$$
\left\{\begin{array}{l}
u^{\prime \prime}(t)+A u(t)=p+f(t), 0<t<1, \\
u^{\prime}(t)+A u(t)=p+g(t),-1<t<0 \\
u\left(0^{+}\right)=u\left(0^{-}\right), u^{\prime}\left(0^{+}\right)=u^{\prime}\left(0^{-}\right) \\
u(-1)=\varphi, u(1)=\psi
\end{array}\right.
$$

in a Hilbert space $L_{2}[-\ell, \ell]$ with the space operator $A=A^{x}$ defined by the formula (5). Here, $f(t)=f(t, x)$ and $g(t)=g(t, x)$ are given abstract functions, 
$u(t)=u(t, x)$ is unknown function and $p=p(x)$ is the unknown element of $L_{2}[-\ell, \ell]$. The proof of Theorem 2 is based on the theorem on stability of the identification problem (7) (see [2]), the self-adjointness and positive definiteness of the space operator $A^{x}$ defined by formula (5) (see [6]).

\section{Stability of Difference Schemes}

The development and implementation of stable numerical methods for solving the problems at hand are crucial for practical reasons since the analytical solutions are not available most of the time.

In this section, we construct and analyse the first order of accuracy stable difference schemes for the approximate solutions of the nonlocal boundary value problem (1) and the space-wise dependent source identification problem (6). The discretization of these problems is carried out in two steps. In the first step, the spatial discretization is conducted. We define the grid space

$$
[-\ell, \ell]_{h}=\left\{x=x_{n} \mid x_{n}=n h,-M \leq n \leq M, M h=\ell\right\} .
$$

We introduce the Hilbert space $L_{2 h}=L_{2}\left([-\ell, \ell]_{h}\right)$ of the grid functions $\varphi^{h}(x)=$ $\left\{\varphi^{n}\right\}_{-M}^{M}$ defined on $[-\ell, \ell]_{h}$, equipped with the norm

$$
\left\|\varphi^{h}\right\|_{L_{2 h}}=\left(\sum_{x \in[-\ell, \ell]_{h}}\left|\varphi^{h}(x)\right|^{2} h\right)^{1 / 2} .
$$

To the differential operator $A^{x}$ defined by the formula (5), we assign the difference operator $A_{h}^{x}$ by the formula

$$
A_{h}^{x} \varphi^{h}(x)=\left\{-\left(a(x) \varphi \frac{n}{x}\right)_{x}-\beta\left(a(-x) \varphi_{\bar{x}}^{-n}\right)_{x}+\delta \varphi^{n}\right\}_{-M+1}^{M-1},
$$

acting in the space of grid functions $\varphi^{h}(x)=\left\{\varphi^{n}\right\}_{-M}^{M}$ and satisfying the conditions $\varphi_{-M}=\varphi_{-M+1}, \varphi_{M}=\varphi_{M-1}$. Here

$$
\begin{aligned}
& \varphi_{\bar{x}}^{n}=\frac{\varphi^{n}-\varphi^{n-1}}{h}, \quad-M+1 \leq n \leq M, \\
& \varphi_{x}^{n}=\frac{\varphi^{n+1}-\varphi^{n}}{h}, \quad-M \leq n \leq M-1 .
\end{aligned}
$$

It is known that under the assumption (2) the difference operator $A_{h}^{x}$, defined by (8), is a self-adjoint positive definite operator in $L_{2 h}$. Using $A_{h}^{x}$, the first 
discretization step for problems (1) and (6) results in the nonlocal boundary value problem

$$
\left\{\begin{array}{l}
u_{t t}^{h}(t, x)+A_{h}^{x} u^{h}(t, x)=f^{h}(t, x), 0<t<1, \\
u_{t}^{h}(t, x)+A_{h}^{x} u^{h}(t, x)=g^{h}(t, x),-1<t<0, \\
u^{h}\left(0^{+}, x\right)=u^{h}\left(0^{-}, x\right), u_{t}^{h}\left(0^{+}, x\right)=u_{t}^{h}\left(0^{-}, x\right), \\
u^{h}(-1, x)=\sum_{j=1}^{P} \alpha_{j} u^{h}\left(\lambda_{j}, x\right)+\varphi^{h}(x)
\end{array}\right.
$$

and the identification problem

$$
\left\{\begin{array}{l}
u_{t t}^{h}(t, x)+A_{h}^{x} u^{h}(t, x)=p^{h}(x)+f^{h}(t, x), 0<t<1, \\
u_{t}^{h}(t, x)+A_{h}^{x} u^{h}(t, x)=p^{h}(x)+g^{h}(t, x),-1<t<0, \\
u^{h}\left(0^{+}, x\right)=u^{h}\left(0^{-}, x\right), u_{t}^{h}\left(0^{+}, x\right)=u_{t}^{h}\left(0^{-}, x\right), \\
u^{h}(-1, x)=\varphi^{h}(x), u^{h}(1, x)=\psi^{h}(x),
\end{array}\right.
$$

respectively. Here and in the rest of this section, $x \in[-\ell, \ell]_{h}$.

Let $\tau=\frac{1}{N}$ and $t_{k}=k \tau,-N \leq k \leq N$. In the second discretization step, we replace the problems (9) and (10) with the following first order of accuracy difference schemes

$$
\left\{\begin{array}{l}
\frac{u_{k+1}^{h}(x)-2 u_{k}^{h}(x)+u_{k-1}^{h}(x)}{\tau^{2}}+A_{h} u_{k+1}^{h}(x)=f_{k}^{h}(x), \\
f_{k}^{h}(x)=f^{h}\left(t_{k}, x\right), 1 \leq k \leq N-1, \\
\frac{u_{k}^{h}(x)-u_{k-1}^{h}(x)}{\tau}+A_{h} u_{k}^{h}(x)=g_{k}^{h}(x), \\
g_{k}^{h}(x)=g^{h}\left(t_{k}, x\right),-N+1 \leq k \leq 0, \\
u_{1}^{h}(x)-u_{0}^{h}(x)=u_{0}^{h}(x)-u_{-1}^{h}(x), \\
u_{-N}^{h}(x)=\sum_{j=1}^{P} \alpha_{j} u_{\left[\frac{\lambda_{j}}{\tau}\right]}^{h}(x)+\varphi^{h}(x),
\end{array}\right.
$$




$$
\left\{\begin{array}{l}
\frac{u_{k+1}^{h}(x)-2 u_{k}^{h}(x)+u_{k-1}^{h}(x)}{\tau^{2}}+A_{h}^{x} u_{k+1}^{h}(x)=p^{h}(x)+f_{k}^{h}(x), \\
f_{k}^{h}(x)=f^{h}\left(t_{k}, x\right), 1 \leq k \leq N-1, \\
\frac{u_{k}^{h}(x)-u_{k-1}^{h}(x)}{\tau}+A_{h}^{x} u_{k}^{h}(x)=p^{h}(x)+g_{k}^{h}(x), \\
g_{k}^{h}(x)=g^{h}\left(t_{k}, x\right),-N+1 \leq k \leq 0, \\
u_{1}^{h}(x)-u_{0}^{h}(x)=u_{0}^{h}(x)-u_{-1}^{h}(x), \\
u_{-N}^{h}(x)=\varphi^{h}(x), u_{N}^{h}(x)=\psi^{h}(x),
\end{array}\right.
$$

respectively.

Theorem 3. Let $\tau$ and $h$ be sufficiently small numbers. For the solution $\left\{u_{k}^{h}(x)\right\}_{-N}^{N}$ of difference problem (11) the following stability estimates hold

$$
\begin{aligned}
& \max _{-N \leq k \leq N}\left\|u_{k}\right\|_{L_{2 h}} \\
& \leq M_{5}(\delta)\left[\left\|\varphi^{h}\right\|_{L_{2 h}}+\max _{-N+1 \leq k \leq 0}\left\|g_{k}^{h}\right\|_{L_{2 h}}+\max _{1 \leq k \leq N-1}\left\|f_{k}^{h}\right\|_{L_{2 h}}\right], \\
& \max _{1 \leq k \leq N-1}\left\|\frac{u_{k+1}^{h}-2 u_{k}^{h}+u_{k-1}^{h}}{\tau^{2}}\right\|_{L_{2 h}}+\max _{-N+1 \leq k \leq 0}\left\|\frac{u_{k}^{h}-u_{k-1}^{h}}{\tau}\right\|_{L_{2 h}} \\
& +\max _{-N \leq k \leq N}\left\|u_{k}^{h}\right\|_{W_{2 h}^{2}} \leq M_{6}(\delta)\left[\left\|\varphi^{h}\right\|_{W_{2 h}^{2}}+\left\|g_{0}^{h}\right\|_{L_{2 h}}\right. \\
& \left.+\max _{-N+2 \leq k \leq 0}\left\|\frac{g_{k}^{h}-g_{k-1}^{h}}{\tau}\right\|_{L_{2 h}}+\left\|f_{1}^{h}\right\|_{L_{2 h}}+\max _{2 \leq k \leq N-1}\left\|\frac{f_{k}^{h}-f_{k-1}^{h}}{\tau}\right\|_{L_{2 h}}\right] .
\end{aligned}
$$

Proof. Difference scheme (11) can be written as the following abstract difference scheme

$$
\left\{\begin{array}{l}
\frac{u_{k+1}^{h}-2 u_{k}^{h}+u_{k-1}^{h}}{\tau^{2}}+A_{h} u_{k+1}^{h}=f_{k}^{h}, 1 \leq k \leq N-1, \\
\frac{u_{k}^{h}-u_{k-1}^{h}}{\tau}+A_{h} u_{k}^{h}=g_{k}^{h}, \quad-N+1 \leq k \leq 0 \\
u_{1}^{h}-u_{0}^{h}=u_{0}^{h}-u_{-1}^{h}, u_{-N}^{h}=\sum_{j=1}^{P} \alpha_{j} u_{\left[\frac{\lambda_{j}}{\tau}\right]}^{h}+\varphi^{h}
\end{array}\right.
$$

in a Hilbert space $L_{2 h}$ with operator $A_{h}=A_{h}^{x}$ defined by formula (8). Here, $f_{k}^{h}=f_{k}^{h}(x)$ and $g_{k}^{h}=g_{k}^{h}(x)$ are given abstract functions, $u_{k}^{h}=u_{k}^{h}(x)$ is unknown mesh function. The proof of Theorem 3 is based on the stability of the difference scheme (13) (see [4]), the self-adjointness and positive definiteness of the space operator $A_{h}$ in $L_{2 h}$ (see [8]). 
Theorem 4. Let $\tau$ and $h$ be sufficiently small numbers. For the solution $\left\{\left\{u_{k}^{h}(x)\right\}_{-N}^{N}, p^{h}(x)\right\}$ of problem (12) the following stability estimates hold

$$
\begin{gathered}
\max _{-N \leq k \leq N}\left\|u_{k}\right\|_{L_{2 h}}+\left\|\left(A_{h}^{x}\right)^{-1} p^{h}\right\|_{L_{2 h}} \leq M_{7}(\delta)\left[\left\|\varphi^{h}\right\|_{L_{2 h}}+\left\|\psi^{h}\right\|_{L_{2 h}}\right. \\
\left.+\max _{-N+1 \leq k \leq 0}\left\|g_{k}^{h}\right\|_{L_{2 h}}+\max _{1 \leq k \leq N-1}\left\|f_{k}^{h}\right\|_{L_{2 h}}\right], \\
\max _{1 \leq k \leq N-1}\left\|\frac{u_{k+1}^{h}-2 u_{k}^{h}+u_{k-1}^{h}}{\tau^{2}}\right\|\left\|_{L_{2 h}}+\max _{-N+1 \leq k \leq 0}\right\| \frac{u_{k}^{h}-u_{k-1}^{h}}{\tau}\left\|_{L_{2 h}}+\right\| p^{h} \|_{L_{2 h}} \\
+\max _{-N \leq k \leq N}\left\|u_{k}^{h}\right\|_{W_{2 h}^{2}} \leq M_{8}(\delta)\left[\left\|\varphi^{h}\right\|_{W_{2 h}^{2}}+\left\|\psi^{h}\right\|_{W_{2 h}^{2}}+\left\|g_{0}^{h}\right\|_{L_{2 h}}\right. \\
\left.+\max _{-N+2 \leq k \leq 0}\left\|\frac{g_{k}^{h}-g_{k-1}^{h}}{\tau}\right\|_{L_{2 h}}+\left\|f_{1}^{h}\right\|_{L_{2 h}}+\max _{2 \leq k \leq N-1}\left\|\frac{f_{k}^{h}-f_{k-1}^{h}}{\tau}\right\|_{L_{2 h}}\right] .
\end{gathered}
$$

Proof. Difference scheme (12) can be written in the following abstract form

$$
\left\{\begin{array}{l}
\frac{u_{k+1}^{h}-2 u_{k}^{h}+u_{k-1}^{h}}{\tau^{2}}+A_{h} u_{k+1}^{h}=p^{h}+f_{k}^{h}, 1 \leq k \leq N-1, \\
\frac{u_{k}^{h}-u_{k-1}^{h}}{\tau}+A_{h} u_{k}^{h}=p^{h}+g_{k}^{h}, \quad-N+1 \leq k \leq 0, \\
u_{1}^{h}-u_{0}^{h}=u_{0}^{h}-u_{-1}^{h}, u_{-N}^{h}=\varphi^{h}, u_{N}^{h}=\psi^{h}
\end{array}\right.
$$

in a Hilbert space $L_{2 h}$ with operator $A_{h}=A_{h}^{x}$ defined by formula (8). Here, $f_{k}^{h}=f_{k}^{h}(x)$ and $g_{k}^{h}=g_{k}^{h}(x)$ are given abstract functions, $u_{k}^{h}=u_{k}^{h}(x)$ is unknown mesh function and $p^{h}=p^{h}(x)$ is the unknown mesh element of $L_{2 h}$. The proof of Theorem 4 is based on the stability of the difference scheme (14) (see [11]), the self-adjointness and positive definiteness of the space operator $A_{h}$ in $L_{2 h}$ (see $[8])$.

\section{Numerical Examples}

In this section, we illustrate how the constructed first order of accuracy difference schemes can be applied for two test problems. The numerical algorithms for implementing these difference schemes are described in [1] and based on a procedure of modified Gauss elimination method [17]. Through the error analysis, we show the convergence of the first order of accuracy difference schemes. 
First, we consider the following nonlocal problem

$$
\left\{\begin{array}{l}
u_{t t}(t, x)-u_{x x}(t, x)-0.5\left(u_{x}(t,-x)\right)_{x}+u(t, x) \\
\quad=f(t, x), x \in(-\pi, \pi), t \in(0,1) \\
\quad u_{t}(t, x)-u_{x x}(t, x)-0.5\left(u_{x}(t,-x)\right)_{x}+u(t, x) \\
\quad=g(t, x), x \in(-\pi, \pi), t \in(-1,0) \\
u\left(0^{+}, x\right)=u\left(0^{-}, x\right), u_{t}\left(0^{+}, x\right)=u_{t}\left(0^{-}, x\right), x \in[-\pi, \pi] \\
u(-1, x)=u(1, x), x \in[-\pi, \pi] \\
u_{x}(t,-\pi)=u_{x}(t, \pi)=0, t \in[-1,1]
\end{array}\right.
$$

for one-dimensional hyperbolic-parabolic equation with involution and Neumann boundary condition, where

$$
\begin{aligned}
& f(t, x)=1.5 \cos t \cos x, x \in(-\pi, \pi), t \in(0,1), \\
& g(t, x)=(2.5 \cos t-\sin t) \cos x, x \in(-\pi, \pi), t \in(-1,0) .
\end{aligned}
$$

The exact solution of problem (15) is given by

$$
u(t, x)=\cos t \cos x, \quad-\pi \leq x \leq \pi, \quad-1 \leq t \leq 1 \text {. }
$$

Let $\tau=\frac{1}{N}$ and $h=\frac{\pi}{M}$. We define the set of grid points as following

$$
\left\{\left(t_{k}, x_{n}\right) \mid t_{k}=k \tau,-N \leq k \leq N, x_{n}=n h,-M \leq n \leq M\right\} .
$$

For the numerical solution of problem (15), we construct the first order of accuracy difference scheme in $t$

$$
\left\{\begin{array}{l}
\frac{u_{n}^{k+1}-2 u_{n}^{k}+u_{n}^{k-1}}{\tau^{2}}-\frac{u_{n+1}^{k+1}-2 u_{n}^{k+1}+u_{n-1}^{k+1}}{h^{2}}-\frac{u_{-n+1}^{k+1}-2 u_{-n}^{k+1}+u_{-n-1}^{k+1}}{2 h^{2}}+u_{n}^{k+1} \\
\quad=f\left(t_{k}, x_{n}\right), 1 \leq k \leq N-1,-M+1 \leq n \leq M-1, \\
\frac{u_{n}^{k}-u_{n}^{k-1}}{\tau}-\frac{u_{n+1}^{k}-2 u_{n}^{k}+u_{n-1}^{k}}{h^{2}}-\frac{u_{-n+1}^{k}-2 u_{-n}^{k}+u_{-n-1}^{k}}{2 h^{2}}+u_{n}^{k} \\
\quad=g\left(t_{k}, x_{n}\right),-N+1 \leq k \leq 0,-M+1 \leq n \leq M-1, \\
u_{n}^{1}-u_{n}^{0}=u_{n}^{0}-u_{n}^{-1}, u_{n}^{-N}=u_{n}^{N}, \quad-M \leq n \leq M, \\
u_{-M}^{k}=u_{-M+1}^{k}, u_{M}^{k}=u_{M-1}^{k},-N \leq k \leq N .
\end{array}\right.
$$

The numerical solutions of this difference scheme are computed for different values of $M$ and $N$. We measure the error between the exact and numerical 
solutions by

$$
\left\|E_{u}\right\|_{\infty}=\max _{\substack{-N \leq k \leq N \\-M \leq n \leq M}}\left|u\left(t_{k}, x_{n}\right)-u_{n}^{k}\right|,
$$

where $u\left(t_{k}, x_{n}\right)$ is the exact value of $u(t, x)$ at $\left(t_{k}, x_{n}\right)$ and $u_{n}^{k}$ represents the corresponding numerical solution. Table 1 shows the errors between the exact solution of the problem (15) and the numerical solutions of the first order of accuracy difference scheme.

Table 1: The errors of the numerical solutions of the first order of accuracy difference scheme for the problem (15).

\begin{tabular}{|l|c|c|}
\hline & $\left\|E_{u}\right\|_{\infty}$ & Order \\
\hline$N=M=20$ & $1.020 \times 10^{-1}$ & - \\
\hline$N=M=40$ & $4.975 \times 10^{-2}$ & 1.036 \\
\hline$N=M=80$ & $2.457 \times 10^{-2}$ & 1.018 \\
\hline$N=M=160$ & $1.221 \times 10^{-2}$ & 1.009 \\
\hline$N=M=320$ & $6.084 \times 10^{-3}$ & 1.005 \\
\hline
\end{tabular}

Second, we consider the following source identification problem

$$
\left\{\begin{array}{l}
u_{t t}(t, x)-u_{x x}(t, x)-0.5\left(u_{x}(t,-x)\right)_{x}+u(t, x) \\
=p(x)+f(t, x), x \in(-\pi, \pi), t \in(0,1), \\
u_{t}(t, x)-u_{x x}(t, x)-0.5\left(u_{x}(t,-x)\right)_{x}+u(t, x) \\
=p(x)+g(t, x), x \in(-\pi, \pi), t \in(-1,0), \\
u\left(0^{+}, x\right)=u\left(0^{-}, x\right), u_{t}\left(0^{+}, x\right)=u_{t}\left(0^{-}, x\right), x \in[-\pi, \pi], \\
u(-1, x)=\varphi(x), u(1, x)=\psi(x), x \in[-\pi, \pi], \\
u_{x}(t,-\pi)=u_{x}(t, \pi)=0, t \in[-1,1]
\end{array}\right.
$$

for one-dimensional hyperbolic-parabolic equation with involution and Neumann boundary condition, where

$$
\begin{aligned}
& f(t, x)=(1.5 \cos t-1) \cos x, x \in(-\pi, \pi), t \in(0,1), \\
& g(t, x)=(2.5 \cos t-\sin t-1) \cos x, x \in(-\pi, \pi), t \in(-1,0), \\
& \varphi(x)=\cos 1 \cos x, \psi(x)=\cos 1 \cos x, x \in[-\pi, \pi] .
\end{aligned}
$$


The exact solution of problem (16) is the pair of functions

$$
(u(t, x), p(x))=(\cos t \cos x, \cos x), \quad-\pi \leq x \leq \pi, \quad-1 \leq t \leq 1 .
$$

For the numerical solution of source identification problem (16), we construct the first order of accuracy difference scheme in $t$

$$
\left\{\begin{array}{l}
\frac{u_{n}^{k+1}-2 u_{n}^{k}+u_{n}^{k-1}}{\tau^{2}}-\frac{u_{n+1}^{k+1}-2 u_{n}^{k+1}+u_{n-1}^{k+1}}{h^{2}}-\frac{u_{-n+1}^{k+1}-2 u_{-n}^{k+1}+u_{-n-1}^{k+1}}{2 h^{2}}+u_{n}^{k+1} \\
\quad=p_{n}+f\left(t_{k}, x_{n}\right), 1 \leq k \leq N-1,-M+1 \leq n \leq M-1, \\
\frac{u_{n}^{k}-u_{n}^{k-1}}{\tau}-\frac{u_{n+1}^{k}-2 u_{n}^{k}+u_{n-1}^{k}}{h^{2}}-\frac{u_{-n+1}^{k}-2 u_{-n}^{k}+u_{-n-1}^{k}}{2 h^{2}}+u_{n}^{k} \\
\quad=p_{n}+g\left(t_{k}, x_{n}\right),-N+1 \leq k \leq 0,-M+1 \leq n \leq M-1, \\
u_{n}^{1}-u_{n}^{0}=u_{n}^{0}-u_{n}^{-1}, u_{n}^{-N}=\varphi\left(x_{n}\right), u_{n}^{N}=\psi\left(x_{n}\right),-M \leq n \leq M, \\
u_{-M}^{k}=u_{-M+1}^{k}, u_{M}^{k}=u_{M-1}^{k},-N \leq k \leq N,
\end{array}\right.
$$

where $u_{n}^{k}$ and $p_{n}$ denote the numerical approximations of $u(t, x)$ at $(t, x)=$ $\left(t_{k}, x_{n}\right)$ and $p(x)$ at $x=x_{n}$, respectively. The numerical solutions of this difference scheme are computed for different values of $M$ and $N$. Table 2 shows the errors between the exact solution of the problem (16) and the numerical solutions of the first order of accuracy scheme. We observe that the scheme has the first order convergence as it is expected to be.

Table 2: The errors of the numerical solutions of the first order of accuracy difference scheme for the problem (16).

\begin{tabular}{|l|c|c|c|c|}
\hline & $\left\|E_{p}\right\|_{\infty}$ & Order & $\left\|E_{u}\right\|_{\infty}$ & Order \\
\hline$N=M=20$ & $9.787 \times 10^{-2}$ & - & $5.229 \times 10^{-2}$ & - \\
\hline$N=M=40$ & $4.927 \times 10^{-2}$ & 0.990 & $2.591 \times 10^{-2}$ & 1.013 \\
\hline$N=M=80$ & $2.472 \times 10^{-2}$ & 0.995 & $1.289 \times 10^{-2}$ & 1.007 \\
\hline$N=M=160$ & $1.238 \times 10^{-2}$ & 0.997 & $6.428 \times 10^{-3}$ & 1.004 \\
\hline$N=M=320$ & $6.198 \times 10^{-3}$ & 0.999 & $3.210 \times 10^{-3}$ & 1.002 \\
\hline
\end{tabular}




\section{References}

[1] M. Ashyraliyev, M.A. Ashyralyyeva, A. Ashyralyev, A note on the hyperbolic-parabolic identification problem with involution and Dirichlet boundary condition, Bull. of the Karaganda University-Mathematics, 99 (2020), 120-129.

[2] A. Ashyralyev, M.A. Ashyralyyeva, On source identification problem for a hyperbolic-parabolic equation, Contemp. Anal. Appl. Math., 3 (2015), 88-103.

[3] A. Ashyralyev, Y. Ozdemir, On nonlocal boundary value problems for hyperbolic-parabolic equations, Taiwanese J. Math., 11 (2007), 1075-1089.

[4] A. Ashyralyev, Y. Ozdemir, On numerical solutions for hyperbolicparabolic equations with the multipoint nonlocal boundary condition, $J$. Franklin Inst., 351 (2014), 602-630.

[5] A. Ashyralyev, A.M. Sarsenbi, Well-posedness of an elliptic equation with involution, Electron. J. Differential Equations, 2015, No 284 (2015), 1-8.

[6] A. Ashyralyev, A.M. Sarsenbi, Stability of a hyperbolic equation with the involution, In: Springer Proc. Math. Stat. of Intern. Conf. on Funct. Anal. in Interdiscip. Appl., Astana, Kazakhstan, Oct. 2-5, 2017 (Ed. by: M.A. Sadybekov and others), Springer, New York (2017), 216, 204-212.

[7] A. Ashyralyev, A. Sarsenbi, Well-posedness of a parabolic equation with involution, Numer. Funct. Anal. Optim., 38, No 10 (2017), 1295-1304.

[8] A. Ashyralyev, P.E. Sobolevskii, New Difference Schemes for Partial Differential Equations, Operator Theory Advances and Applications, Birkhauser Verlag, Basel (2004).

[9] M. Ashyralyyeva, M. Ashyraliyev, Numerical solutions of source identification problem for hyperbolic-parabolic equations, In: AIP Conf. Proc. 1997 (4th Intern. Conf. on Anal. and Appl. Math., Mersin, Turkey, September 69, 2018) (Ed. by: A. Ashyralyev and others), American Institute of Physics, New York (2018), Art. 020048.

[10] M.A. Ashyralyyeva, M. Ashyraliyev, On the numerical solution of identification hyperbolic-parabolic problems with the Neumann boundary condition, Bull. of the Karaganda University-Mathematics, 91 (2018), 69-74. 
[11] M.A. Ashyralyyeva, A. Ashyralyyev, Stable difference scheme for the solution of the source identification problem for hyperbolic-parabolic equations, In: AIP Conf. Proc. 1676 (Intern. Conf. on Adv. in Math. Sci., Antalya, Turkey, Nov. 5-7, 2015)(Ed. by: A. Ashyralyev and others), American Institute of Physics, New York (2015), Art. 020024.

[12] A.V. Bitsadze, Equations of Mixed Type, Pergamon (1964).

[13] A. Cabada, F. Tojo, F. Adrian, Differential Equations with Involutions, Atlantis Press (2015).

[14] S.I. Kabanikhin, Inverse and Ill-Posed Problems: Theory and Applications, De Gruyter (2011).

[15] A.I. Prilepko, D.G. Orlovsky, I.A. Vasin, Methods for Solving Inverse Problems in Mathematical Physics, Marcel Dekker (2000).

[16] J.M. Rassias, Lecture Notes on Mixed Type Partial Differential Equations, World Scientific (1990).

[17] A.A. Samarskii, E.S. Nikolaev, Numerical Methods for Grid Equations: Iterative Methods, Birkhauser Verlag, Basel (1989).

[18] M.M. Smirnov, Equations of Mixed Type, American Mathematical Society (1978). 